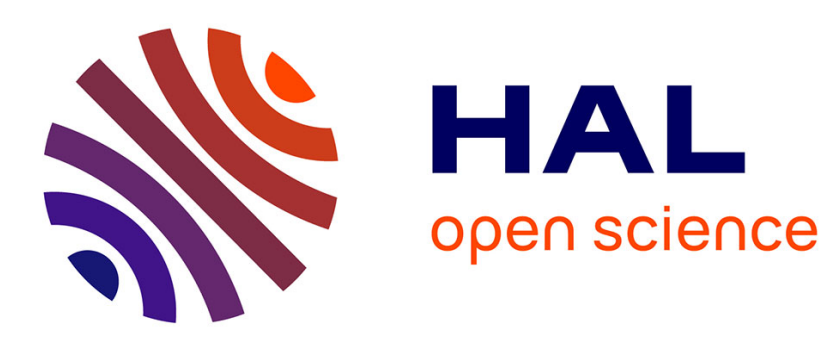

\title{
Generalized finite difference scheme using mainly orthogonal and locally barycentric dual mesh for electromagnetic problems
}

\author{
L. Bernard, L. Pichon
}

\section{- To cite this version:}

L. Bernard, L. Pichon. Generalized finite difference scheme using mainly orthogonal and locally barycentric dual mesh for electromagnetic problems. European Physical Journal: Applied Physics, 2010, 52 (2), 10.1051/epjap/2010081 . hal-00634367

\section{HAL Id: hal-00634367 https://hal.science/hal-00634367}

Submitted on 21 Oct 2011

HAL is a multi-disciplinary open access archive for the deposit and dissemination of scientific research documents, whether they are published or not. The documents may come from teaching and research institutions in France or abroad, or from public or private research centers.
L'archive ouverte pluridisciplinaire HAL, est destinée au dépôt et à la diffusion de documents scientifiques de niveau recherche, publiés ou non, émanant des établissements d'enseignement et de recherche français ou étrangers, des laboratoires publics ou privés. 


\title{
Generalized Finite Difference Scheme Using Mainly Orthogonal and Locally Barycentric Dual Mesh for Electromagnetic Problems
}

\author{
L. Bernard and L. Pichon
}

Laboratoire de Génie Electrique de Paris, CNRS UMR 8507; SUPELEC; UPMC Univ Paris-Sud; 11 rue Joliot-Curie, Plateau de Moulon, F-91192 Gif-sur-Yvette Cedex.

Received: date / Revised version: date

\begin{abstract}
A mainly orthogonal and locally barycentric dual mesh is used to improve the performances of a generalized finite difference method. A criterium is proposed to choose between an orthogonal and a barycentric construction for the dual mesh taking into account stability considerations for an explicit time scheme. The construction of the constitutive matrix is performed using either the Microcell or the Galerkin method. The proposed method is shown to considerably reduce the computational cost in the assembly process and the resolution compared to methods using completely barycentric dual meshes.
\end{abstract}

PACS. 02.70.Bf Finite-difference methods - 02.70.Dh Finite-element and Galerkin methods - 41.20.Jb

Electromagnetic wave propagation; radiowave propagation

\section{Introduction}

The resolution of complex electromagnetic problems requires the use of numerical methods. Among the mesh based methods, largely used ones consist in integrating Maxwell equations on the faces of a complex of primal and dual meshes: in the original FIT (Finite Integration Technique), the meshes are made of regular hexahedral cells [1]; the Microcell method was first developed on meshes composed of normally extruded triangles [2]. These meth- ods can also be generalized to use other types of cells and the respective theory is developped in the Cell Method [3] and the Generalized Finite Difference method [4] which give general tools for the treatment of partial differential equations:

- when applied to regular hexahedral meshes with an explicit time scheme, they are equivalent to the FDTD (Finite Difference Time Domain method), 
- when using a barycentric dual mesh and special constitutive matrices, they are equivalent to the Finite Element Method.

Recent investigations are mainly focused on the choice of the electromagnetic quantities to discretize on the primal and the dual meshes [5], and on the construction of the constitutive matrices [6] [7]. In fact, the characteristics of the meshes and of the constitutive matrices are crucial. For example, when the primal and the dual meshes are orthogonal, the constitutive matrices are diagonal and the efficiency of the computation is optimal. Unfortunately, the construction of a complex of orthogonal meshes is not always possible, and the constitutive matrices have to be built using specific methods. The two options studied here are:

- the Microcell method, which can be applied to any type of dual mesh,

- the Galerkin method with Whitney elements, which can only be applied with a barycentric dual mesh.

The aim of the proposed method is to take advantage of the orthogonal dual mesh wherever possible. The method is developped in a 2-dimensional case using an unstructured triangular primal mesh. A criterium is proposed in order to choose between circumcenters and barycenters for the nodes of the dual mesh. The construction of the constitutive matrices is performed using the Microcell or the Galerkin method. Numerical results are analysed for a simple problem: a plane electromagnetic pulse propagating in a waveguide.

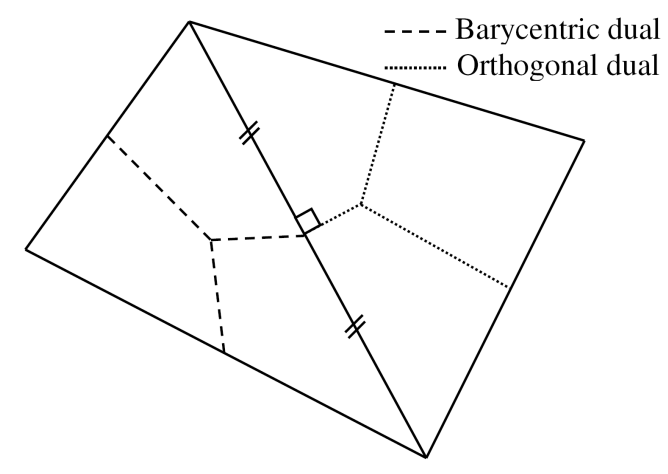

Fig. 1. Connection between orthogonal and barycentric dual meshes

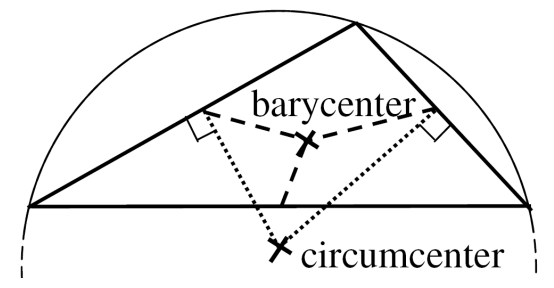

Fig. 2. Cell that do not contain its circumcenter

\section{Dual mesh}

The primal mesh is unstructured and consists of triangular cells. There are mainly two interesting ways to construct the dual mesh:

- the orthogonal construction (Voronoï diagram) in which dual nodes are the circumcenters of the primal cells and dual edges are segments of the perpendicular bisectors of the primal edges,

- the barycentric construction in which dual nodes are the barycenters of the primal cells and dual edges consists of two segments joining the dual nodes to the barycenters of the primal edges.

In the 2-dimensional case, barycentric and orthogonal constructions of the dual mesh naturally connect at the middle of a primal edge (Fig. 1). 


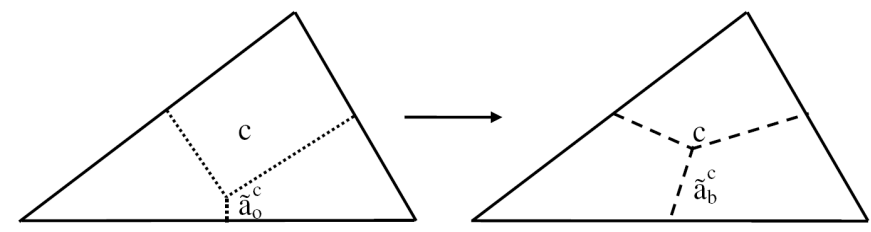

Fig. 3. Left: orthogonal construction with small dual edge; Right: better barycentric construction.

If the triangular primal mesh is carefully built, only few cells do not contain their circumcenters (Fig. 2). For these cells, the dual mesh must be built using the barycentric construction. For all the other elements, the orthogonal construction of the dual mesh can be applied. However, some of the primal cells' circumcenters lie near the boundaries so that the respective parts of dual edges are small. When using an explicit time scheme, the stability depends on the time step and the characteristic size of the space discretization, just like it depends on the CFL criterium in the FDTD method. In consequence, a small edge may reduce the maximum time step that ensures stability. In order to prevent this problem, some of the circumcenters must be rejected from the dual mesh (Fig. 3). The stability issue is studied more thoroughly in sections 4 and 5 .

The dual of a primal edge $(a)$ is an edge named $\tilde{a}$. The portion of the dual edge included in a primal cell $(c)$ is denoted $\tilde{a}^{c}$. This notation stands for an edge, for its length or for the vector normal to the edge and which norm is equal to the length of the edge, depending on the context. The subscript 'o' (respectively 'b') is used to indicate that the orthogonal (respectively barycentric) construction of the dual mesh is considered. For each portion of dual edge, the ratio $\mathrm{r}$ is computed:

$$
r=\frac{\tilde{a}_{o}^{c}}{\tilde{a}_{b}^{c}}
$$

Whenever $r<0.3$, the barycentric construction is used for the cell containing the considered portion of dual edge. This criterium will be discussed in the numerical results section 5 .

\section{Construction of the constitutive matrices}

The vectors containing the unknowns of the 2-dimensional problem are:

$-e$, circulations of the electric field $(E)$ on the edges $(a)$ of the primal mesh,

$-d$, fluxes of the electric displacement $(D)$ through the edges $(\tilde{a})$ of the dual mesh considered as faces of unit depth,

$-h$, circulations of the magnetic field $(H)$ on unit segments normally crossing the plane at the dual nodes $(\tilde{c})$

$-b$, fluxes of the magnetic induction $(B)$ through the primal cells $(c)$.

As the magnetic field is supposed to be normal to the plane, the constitutive matrix $M_{\nu}$ such that $h=M_{\nu} b$ is diagonal and its entries can be computed from:

$$
M_{\nu i i}=\nu \frac{\tilde{c}_{i}}{c_{i}}=\nu \frac{1}{c_{i}},
$$

where $c_{i}$ is the surface of the primal face and $\nu$ is the inverse of the magnetic permeability in cell $i$. If the primal 


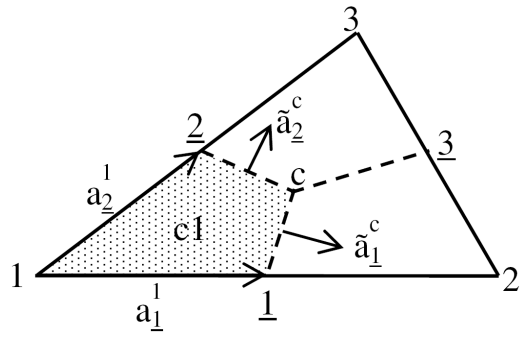

Fig. 4. Microcells and notations for portions of primal and dual edges.

and the dual meshes are not orthogonal, the constitutive matrix $M_{\epsilon}$ such that $d=M_{\epsilon} e$ is not diagonal and must be built using elementary constitutive matrices.

\subsection{Microcell method}

The microcell method was first developped by Marrone [2]. A microcell is this intersection of a primal cell and a dual cell. A primal triangular cell consists of three quadrilateral microcells. The electric field and permittivity $(\epsilon)$ are supposed to be constant on each microcell. For each microcell, partial circulations $\left(e_{\underline{j}}^{i}\right)$ and fluxes $\left(d_{\underline{j}}^{c}\right)$ can be expressed in terms of field components in the xy-plane. For example, in the microcell c1 (intersection of the primal cell c with the dual cell 1, Fig. 4):

$$
\left(\begin{array}{l}
d_{\underline{1}}^{c} \\
d_{\underline{2}}^{c}
\end{array}\right)=\left(\begin{array}{cc}
\tilde{a}_{\underline{1}_{x}}^{c} & \tilde{a}_{\underline{1}_{y}}^{c} \\
\tilde{a}_{\underline{2}_{x}}^{c} & \tilde{a}_{\underline{2}_{y}}^{c}
\end{array}\right)\left(\begin{array}{c}
D_{x} \\
D_{y}
\end{array}\right)
$$

and

$$
\left(\begin{array}{l}
e_{\underline{1}}^{1} \\
e_{\underline{2}}^{1}
\end{array}\right)=\left(\begin{array}{cc}
a_{\underline{1}}^{1} & a_{\underline{1}_{y}}^{1} \\
a_{\underline{2}_{x}}^{1} & a_{\underline{2}_{y}}^{1}
\end{array}\right)\left(\begin{array}{c}
E_{x} \\
E_{y}
\end{array}\right)
$$

where $a_{j}^{i}$ is a vector representing a portion of primal edge, and $\tilde{a}_{j}^{c}$ is the vector normal to the dual edge and which norm is equal to the length of the dual edge. As $D=\epsilon E$, equations (3) and (4) give:

$$
\left(\begin{array}{l}
e_{\underline{1}}^{1} \\
e_{\underline{\underline{2}}}^{1}
\end{array}\right)=\left(\begin{array}{cc}
a_{\underline{1}_{x}}^{1} & a_{\underline{1}_{y}}^{1} \\
a_{\underline{2}_{x}}^{1} & a_{\underline{\underline{2}}_{y}}^{1}
\end{array}\right) \epsilon^{-1}\left(\begin{array}{cc}
\tilde{a}_{\underline{1}_{x}}^{c} & \tilde{a}_{\underline{1}_{y}}^{c} \\
\tilde{a}_{\underline{\underline{2}}_{x}}^{c} & \tilde{a}_{\underline{\underline{2}}_{y}}^{c}
\end{array}\right)^{-1}\left(\begin{array}{l}
d_{\underline{1}}^{c} \\
d_{\underline{\underline{2}}}^{c}
\end{array}\right) .
$$

By writing similar equations for the other microcells of cell c, adding partial circulations considering:

$$
\left\{\begin{array}{l}
e_{\underline{1}}=e_{\underline{1}}^{1}+e_{\underline{1}}^{2} \\
e_{\underline{2}}=e_{\underline{2}}^{1}+e_{\underline{2}}^{3} \\
e_{\underline{3}}=e_{\underline{3}}^{2}+e_{\underline{3}}^{3}
\end{array}\right.
$$

and inverting, an elementary constitutive matrix $m_{\epsilon}$ can be assembled such that:

$$
\left(\begin{array}{l}
d_{\underline{1}}^{c} \\
d_{\underline{2}}^{c} \\
d_{\underline{3}}^{c}
\end{array}\right)=m_{\epsilon}\left(\begin{array}{l}
e_{1} \\
e_{\underline{2}} \\
e_{\underline{3}}
\end{array}\right)
$$

This elementary matrix links the partial fluxes $d^{c}$ to the circulations $e$.

\subsection{Galerkin method}

It has been shown that the Galerkin finite element method is closely related to the generalized finite difference method [8]. Particularly, using Whitney edge elements $(w)$ on the primal triangular mesh, the components of the elementary matrix for the cell c can be computed by:

$$
m_{\epsilon \underline{i j}}=\int_{c} \epsilon w_{\underline{i}} w_{\underline{j}}
$$

The resulting matrix is then symmetric.

\subsection{Assembly}

For all cells having an orthogonal dual, the construction of $M_{\epsilon}$ needs no assembly process. In fact, the diagonal 
entries can be directly computed by:

$$
M_{\epsilon \underline{i}}=\epsilon^{c_{1}} \frac{\tilde{a}_{\underline{i}}^{c_{1}}}{a_{\underline{i}}}+\epsilon^{c_{2}} \frac{\tilde{a}_{\underline{\underline{c}}}^{c_{2}}}{a_{\underline{i}}},
$$

if the edge $\underline{i}$ belongs to two cells $\left(c_{1}\right.$ and $\left.c_{2}\right)$ the dual of which is orthogonal,

$$
M_{\epsilon \underline{i i}}=\epsilon^{c} \frac{\tilde{a}_{\underline{i}}^{c}}{a_{\underline{i}}}
$$

if the edge $\underline{i}$ has an orthogonal dual.

Then the matrix $M_{\epsilon}$ can be completed by applying an assembly procedure to the cells having a barycentric dual, using the elementary constitutive matrices generated either by the microcell method or the Galerkin method.

\section{Time scheme and maximum time step}

A leapfrog scheme is applied to discretize Maxwell's equations in the time domain:

$$
\left\{\begin{array}{l}
d^{k+\frac{1}{2}}=d^{k-\frac{1}{2}}+\delta t R^{t} h^{k} \\
b^{k+1}=b^{k}-\delta t R e^{k+\frac{1}{2}}
\end{array}\right.
$$

where $k$ is the time index, $R$ is the discrete rotational operator on the primal mesh and $R^{t}$ its transpose. Using the constitutive relations and eliminating the magnetic quantities, this system gives a second order recurrence relation:

$$
e^{k+\frac{3}{2}}-\left(2-\delta t^{2} M_{\epsilon}^{-1} R^{t} M_{\nu} R\right) e^{k+\frac{1}{2}}+e^{k-\frac{1}{2}}=0
$$

The analysis of the characteristic equation of this recurrence gives the stability condition [9]:

$$
\delta t<\frac{2}{\sqrt{\lambda_{\max }}}
$$

where $\lambda_{\max }$ is the greatest eigenvalue of the generalized eigenvalue problem:

$$
R^{t} M_{\nu} R v=\lambda M_{\epsilon} v
$$

and $\delta t$ is the time step of the leapfrog scheme.

\section{Numerical results}

A parallel-plate waveguide is considered with perfect magnetic conductor (PMC) boundaries at its terminations. A gaussian pulse is generated at the left end and propagates longitudinally through the guide. The pulse is totally reflected at the PMC and goes without attenuation from one boundary to the other. The primal triangular mesh is generated using Gmsh [10] (Fig. 5), it consists of 2034 cells. Various dual meshes are constructed for values of the parameter $\mathrm{r}$ (1) varying from 0 (maximum number of circumcenters) to 1 (completely barycentric dual). The respective rate of primal cells having a barycentric dual varies from $3 \%$ to $100 \%$ (Fig. 6). For each value of $r$, the constitutive matrices are computed as presented in section 3 using the Galerkin or the Microcell method. The maximum time step is calculated from (13) using an Arnoldi algorithm to solve the eigenvalue problem (14) (Fig. 7). It can be noted that the rate of barycentric dual nodes increases constantly with $\mathrm{r}$ whereas the maximum time step is piecewise constant. The change of a dual node from circumcenter to barycenter does not necessarily implies an increase of the maximum time step. A slight decrease of the maximum time step is observed for $r>0.4$. For the lowest values of $r$, the maximum time step is significantly 


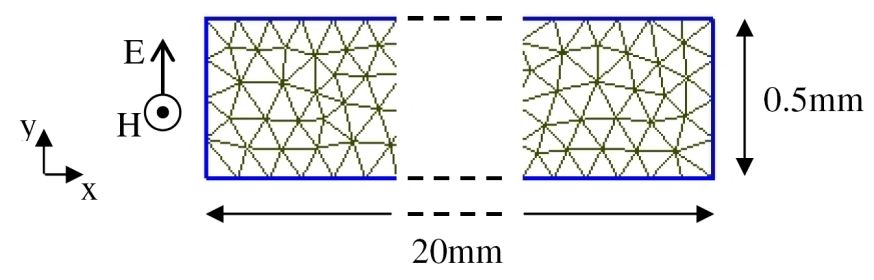

Fig. 5. Waveguide: plane gaussian pulse generated at the left boundary; Left and right boundaries: perfect magnetic conductor; Bottom and top boundaries: perfect electric conductor

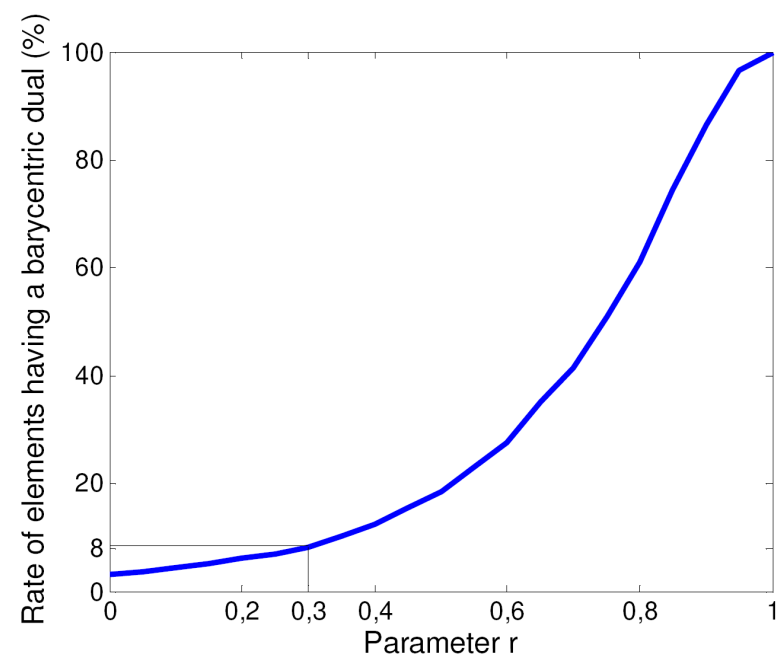

Fig. 6. Rate of elements having a barycentric dual as function of parameter $\mathrm{r}$

lower. $r=0.3$ is then selected for the choice criterium of section 2. In consequence, the dual mesh consists of only $8 \%$ of barycentric nodes.

The time resolution of the problem is then run with a time step $\delta t=0.95 \delta t_{\max }$ for 30000 iterations. The value of the y-component of the electric field is computed at the center of the waveguide and is compared to the analytical solution of the problem (Fig. 7). The proposed method shows stable results and good agreement with the analytical solution even for the last time iterations.

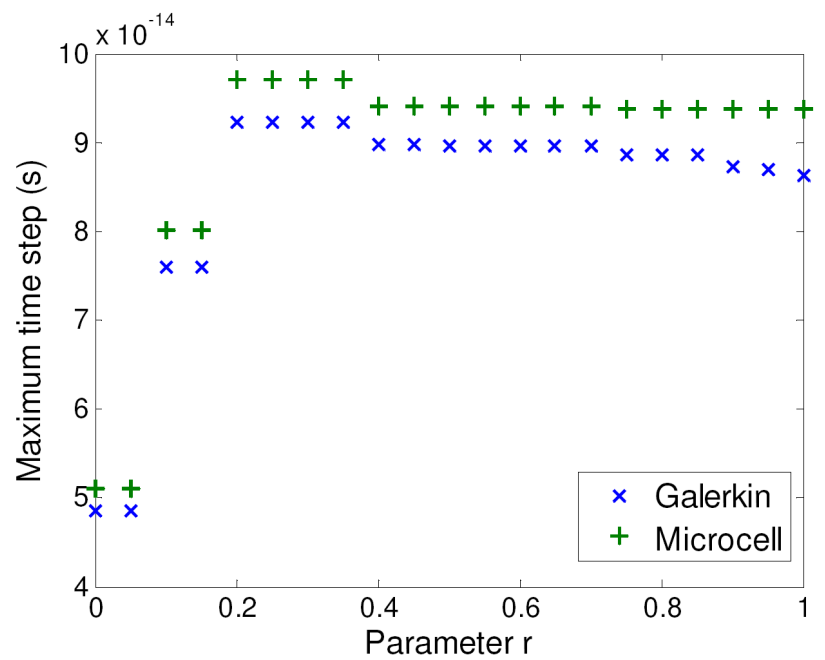

Fig. 7. Maximum time step as function of parameter $r$

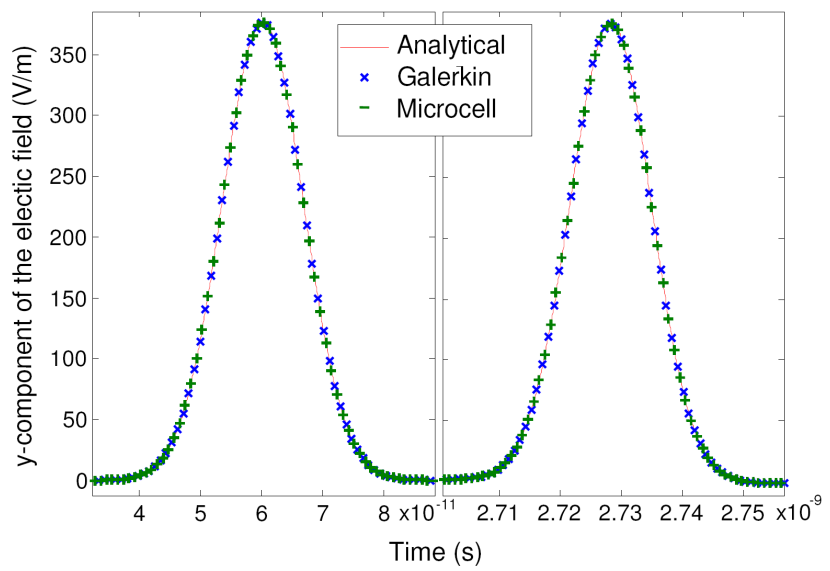

Fig. 8. y-component of the electric field at the center of the guide at first and last times ( 30000 time iterations)

\section{Conclusion}

The use of a mainly orthogonal and locally barycentric dual mesh in a generalized finite difference method allows to work with a triangular unstructured primal mesh and to reduce the computational cost: the assembly process needs to be done only on $8 \%$ of the primal cells and the resulting constitutive matrices are mainly diagonal. It is shown that the method works well using the Galerkin or the Microcell method to compute the elementary constitutive matrices 
in the 2-D case. This work will be extended to the $3-\mathrm{D}$ case taking into acount that orthogonal and barycentric dual meshes do not connect naturally on tetrahedra.

\section{References}

1. T.Weiland, Electronics and Communications 31, 3 (1977) 116-120.

2. M.Marrone, PIER 32, (2001) 317-356.

3. E.Tonti, PIER 32, (2001) 1-44.

4. A.Bossavit, PIER 32, (2001) 45-64.

5. J.Korecki et al., COMPEL 27, 1 (2008) 47-55.

6. P.Alotto et al., IEEE Trans. Mag. 42, 4 (2006) 799-802.

7. B.Auchmann et al., IEEE Trans. Mag. 42, 4 (2006) 643-646.

8. A.Bossavit, J. Japan Soc. Appl. Electromagn. 8, (2000) 203209.

9. Jianming Jin, The finite element method in electromagnetics (Wiley-Interscience, 2002) 536-538.

10. http://www.geuz.org/gmsh/. 